\title{
Penetrated Shotgun Pellets: A Case Report
}

M. Isa Karaa, DDS

Hidayet B. Polat ${ }^{\mathrm{b}}$, DDS, PhD

Sinan $A y^{c}$, DDS, PhD

\section{ABSTRACT}

Shotgun wounds can result in devastating functional and aesthetic consequences for patients. There is no consensus in terms of removing or retaining foreign bodies such as shotgun pellets. In this report a 54-year-old man who suffered from accidental shotgun wounds on the face approximately 26 years ago is presented. Although most of pellets were still present, there were no symptoms such as poisoning, fistula formation, recurrent infections, or secondary hemorrhage to date except feeling cold in cold days. (Eur J Dent 2008;2:59-62)

Key words: Shotgun; Pellet; Shotgun wound.

\section{INTRODUCTION}

Shotgun wounds, especially those on the face are not relatively more frequent in Turkey than some countries such as USA and some Latin American nations due to the fact that it is difficult to obtain firearms legally. ${ }^{1,2}$ But shotguns, generally used for hunting purposes, are common in rural areas and sometimes they are used to commit

\footnotetext{
- a Res Assist. Department of Oral and Maxillofacial Surgery, Cumhuriyet University, Faculty of Dentistry, Sivas, Turkey.

b Res Assist. Department of Oral and Maxillofacial Surgery, Cumhuriyet University, Faculty of Dentistry, Sivas, Turkey. Assoc Prof. Department of Oral and Maxillofacial Surgery, Gaziantep University, Faculty of Dentistry, Gaziantep, Turkey.

- Corresponding Author: Dr. Hidayet B. Polat Department of Oral and Maxillofacial Surgery, Faculty of Dentistry, Cumhuriyet University 58140, Sivas, Turkey Phone: +90 3462191010 / 2752 Fax: +90 3462191237 E-mail: hidayetpolatayahoo.com
}

suicide or to attempt suicide but also at the same time can cause accidental wounds. ${ }^{3}$

The results of multiple shotgun wounds especially in head and neck are fatal. ${ }^{4}$ People who remain alive after multiple injury require the coordination of multiple surgical disciplines to optimize the functional and aesthetic consequence. ${ }^{5}$ Definitive management of patients with shotgun wounds remains controversial in terms of reconstruction of bone and soft tissue defects. ${ }^{6}$ Conventional management of these wounds dictates initial debridement and closure of the soft tissues combined with external fixation of the remaining maxillary and mandibular bone segments. Definitive bone replacement is delayed until soft-tissue replacement is completed. ${ }^{?}$

This case report presents a patient who suffered from accidental shotgun wounds with many penetrated pellets in the maxillofacial region and without any symptoms for 26 years. 


\section{CASE REPORT}

A 54-year-old man applied to our clinic with a complaint of a mobile foreign body in the left maxillary vestibular area. In radiographic examination, many irregular radiopaque penetrated objects were detected bilaterally in the maxillofacial region and it was seen that the periphery of those foreign bodies were walled off with fibrous capsule without any symptoms (Figure 1a, b).

Detailed anamnesis was taken and history revealed that patient suffered from accidental shotgun wounds on his maxillofacial region about 26 years ago. The other medical history was noncontributory. According to what the patient said, he was standing at about 1 meter distance from his cousin who accidentally shot him. Then, he was immediately taken to Local Hospital. At the hospital, after basic resuscitation, accessible pellets were removed but other unattainable pellets were left. Then soft tissue was repaired and exposed wound was closed. After soft tissue healing was achieved, only rhinoplasty operation was performed.

As there were probably a lot of pellets lodged in the maxillofacial complex but functional deficit was not present and due to a combination of difficult access and a close anatomical relationship of the pellet to vital structures, subsequent operation was not considered except rhinoplasty for cosmetic purposes. The patient stated that there was just chill feeling due to penetrating pellets in the cold days.

There was only one significant extra-oral finding on examination about abnormal nose appearance. The patient stated that he had undergone surgery 12 times for reconstruction. The intraoral examination showed a round, mobile object with $0.7 \mathrm{~cm}$ in diameter partly in the maxillary alveolar bone and partly attached to the mucosa. No sign of neurological or functional deficit was detected on both extraoral and intraoral examination.

After local anesthetics administration, small incision on attached mucosa was performed and the pellet was removed. Adjacent tissue of the pellet was also curettaged for histopathological purposes but the result was uneventful. Because all the others were asymptomatic and patient was unwilling for further procedures; no operation was considered for the other pellets in the maxilla. For foreign body reaction could possibly occur, the patient was advised to come to routine control every year, regularly.

\section{DISCUSSION}

Firearm wounds to the maxillofacial complex are uncommon and can result in devastating functional and aesthetic consequences for patients. ${ }^{6.8}$ It can result from suicide, homicide or accident. The majority of patients die due to such injuries but some can survive with excessive serious injuries on the face. ${ }^{9}$ Depending on the type of firearm, the velocity of the projectile, the distance from which the patient is shot and tissue resistance are the factors that determine the severity of injury to the affected person. 8,10

The basic type of firearm wounds are classified into three groups: penetrating, perforating, and avulsive. Low-velocity projectiles can cause penetrating wounds that usually create small entrance and exit wounds. In contrast, highvelocity projectiles can cause perforating wounds that pass right through tissues, exhibiting small entry wounds and comparatively larger exit wounds. Shotgun pellets' traveling is usually at medium velocity and due to their irregular
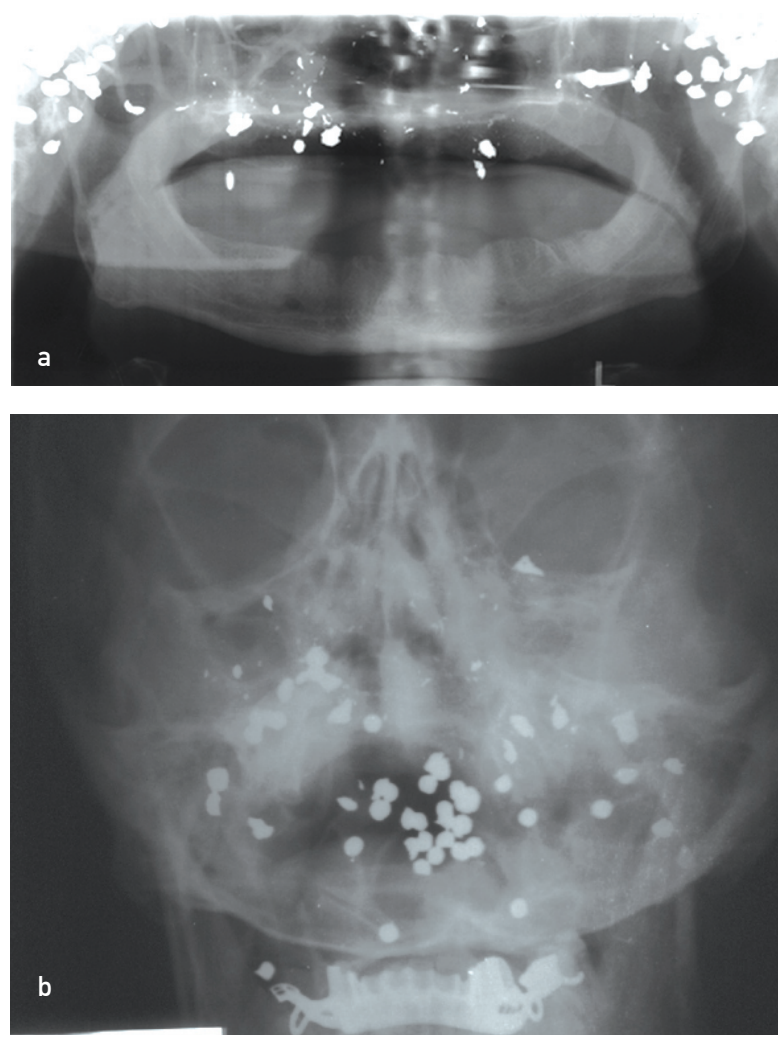

Figure 1a, b. Many of pellets are visible in maxillofacial region without marked sign of pathologic changes. 
fragments they can cause severe hard and soft tissue loss and damage by creating an avulsive wound. Additionally, close-range firearms and high-velocity missile injuries can also create avulsive wound. ${ }^{2}$ In our case, penetrating wounds were caused by shotgun.

Attention may be directed away from these shotgun wounds to the obvious head and neck injury, because failure to detect these injuries may be fatal. ${ }^{8}$ If multiple wounds are caused by shotgun injury, the result generally may be lethal. In contrast; according to Abe et al, ${ }^{4}$ fatal case can be caused by only one shotgun pellet which penetrated the trunk of the victim. In some cases, the pellets caused secondary damage to the victims because of embolization. Although many shotgun pellets were lodged in to maxillofacial region, our patient was lucky and none of pellets did destroy the vital structures, so patient has survived so far.

The emergency care of firearm injuries to the head and neck must be focused on the basics of resuscitation. Primary attention should be given to the condition of airway as bleeding and subsequent swelling can endanger breathing. ${ }^{11}$ After these protocols hemodynamic resuscitation should be performed. ${ }^{8}$ The second step for head and neck injury, detailed examination on vascular and laryngotracheal area must be performed in case of shock, expanding hematoma, active hemorrhage, bruit or thrill, pulse deficit, neurological deficit, subcutaneous emphysema, a "sucking" wound, stridor, dyspnoea, hoarseness, dysphonia or hemoptysis. $^{12}$

Definitive management of patients with shotgun wounds remains controversial in terms of reconstruction of bone and soft tissue defects. ${ }^{6}$ Many authors ${ }^{13,14}$ advocate a non-operative management or conservative approach to just debride the patient in the first operative stage and treat the hard tissues in another operation after soft tissues have been healed and the chance of postoperative infection has been deemed lower. However, some studies ${ }^{7,8}$ advocate early intervention for one-stage management and open treatment of all involved structures. Other series $^{11,15}$ advocate an even more extensive approach, particularly with respect to primary reconstruction of facial soft tissue defects.

As foreign body can cause poisoning, fistula formation, recurrent infections, or secondary hemorrhage, its extraction is recommended even in the absence of clinical symptoms. ${ }^{10}$ When retained bullet causes plumbism, some non-specific symptoms are generally associated with this condition including; anorexia, vomiting, constipation, abdominal pain and occasionally anemia and renal toxicity, but also acute encephalopathy with lethargy, stupor, coma, and convulsions could manifest, in critical cases. ${ }^{16}$ Regardless of velocity, shotgun wounds are evermore contaminated by infectious agents. According to Thorsby and Darlow ${ }^{17}$ bullets inoculated with a heat labile organism fired through sterile nutrient-enriched gelatin will produce bacterial growth along the entire length of the bullet track. In contrast, there are many examples of bullets penetrating deep tissues that remain asymptomatic for the lifetime of the patient. ${ }^{2}$ This case is typical of such injuries; for 26 years pellets lodged at the maxillofacial area have remained asymptomatic. However, relating to atrophied maxillary alveolar bone, one pellet was gradually released after 26 years so it has been interfered with maxillary prosthesis.

In our case, the embedded pellets in the maxillofacial region did not cause any symptoms such as poisoning, fistula formation or infection to date, except feeling cold in the cold days. No secondary symptoms were stated by the patient related to retained pellets. According to these findings, it can be suggested that pellets can retain for long years without any appreciable symptoms, and may left in situ without any intervention, which corroborate some reports of literature.

\section{REFERENCES}

1. Arslan H, Subasi M, Kesemenli C, Kapukaya A, Necmioglu S, Kayikci C. Problem fractures associated with gunshot wounds in children. Injury 2002:33:743-749.

2. Dimitroulis G. An unusual bullet trajectory to the face. $J$ Oral Maxillofac Surg 2006:64:137-139.

3. Suominen E, Tukiainen E. Close-range shotgun and rifle injuries to the face. Clin Plast Surg 2001:28:323-337.

4. Abe M, Motani-Saitoh H, Sato $Y$, Kiuchi M. A fatal case of shotgun injury caused by one pellet. Leg Med 2002:4:131133.

5. Weider L, Hughes K, Ciarochi J, Dunn E. Early versus delayed repair of facial fractures in the multiply injured patient. Am Surg 1999:65:790-793. 
6. Vayvada H, Menderes A, Yilmaz M, Mola F, Kizilkaya A, Atabey A. Management of close-range, high-energy shotgun and rifle wounds to the face. $J$ Craniofac Surg 2005:16:794-804.

7. Gruss JS, Antonyshyn 0, Phillips JH. Early definitive bone and soft-tissue reconstruction of major gunshot wounds of the face. Plast Reconstr Surg 1991:87:436-450.

8. Hollier L, Grantcharova EP, Kattash M. Facial gunshot wounds: a 4-year experience. J Oral Maxillofac Surg 2001:59:277-282

9. Desinan L, Mazzolo GM. Gunshot fatalities: suicide, homicide or accident? A series of 48 cases. Forensic Sci Int 2005:147:37-40.

10. Lee D, Nash M, Turk J, Har-El G. Low-velocity gunshot wounds to the paranasal sinuses. Otolaryngol Head Neck Surg 1997:116:372-378.

11. Motamedi MH. Primary management of maxillofacial hard and soft tissue gunshot and shrapnel injuries. J Oral Maxillofac Surg 2003:61:1390-1398.

12. Holmes JD. Gunshot Injuries. In: Milaro M. ed. Peterson's Principals of Oral and Maxillofacial Surgery. 2nd edn. BC Decker Inc. Hamilton, London, 2004, pp 509-526.

13. Behnia H, Motamedi MH. Reconstruction and rehabilitation of short-range, high-velocity gunshot injury to the lower face: a case report. J Craniomaxillofac Surg 1997:25:220-227.

14. Finn RA. Treatment of comminuted mandibular fractures by closed reduction. J Oral Maxillofac Surg 1996:54:320327.

15. Mladick RA, Georgiade NG, Royer J. Immediate flap reconstruction for massive shotgun wound of face. Plast Reconstr Surg 1970:45:186-188.

16. Mahajan M, Shah N. Accidental lodgment of an air gun pellet in the maxillary sinus of a 6-year old girl: a case report. Dent Traumatol 2004:20:178-180.

17. Thoresby FP, Darlow HM. The mechanisms of primary infection of bullet wounds. Br J Surg 1967:54:359-361. 